\title{
Adenomyotic cyst mimicking a congenital Müllerian anomaly: Diagnosis and treatment with laparoscopy
}

\author{
Sangam Jha \\ Department of Obstetrics and Gynecology, AlIMS Patna, Bihar, India
}

A 28-year-old woman presented with a 1-year history of severe progressive dysmenorrhea following suction evacuation and tubal ligation. Sonography showed a bicornuate uterus with hematometra in the left horn. Hysteroscopy ruled out a diagnosis of a congenital Müllerian anomaly, as both ostia appeared normal. Under laparoscopy, a mass was seen on the left fundal region near the insertion of the round ligament, and needle aspiration of a chocolate-colored fluid confirmed the diagnosis of an adenomyotic cyst. The cyst was excised. The patient recovered well and has been symptom-free since surgery. Adenomyotic cyst is a rare entity in young women and must be differentiated from obstructive Müllerian anomaly. Laparoscopy is the preferred minimally invasive modality for managing this rare disorder.

Keywords: Adenomyotic cyst; Dysmenorrhea; Laparoscopy; Obstructive Müllerian anomaly

\section{Introduction}

Cysts in the myometrium are rare, and the differential diagnosis includes myoma with cystic degeneration, cystic adenomyoma, and obstructive Müllerian anomalies. Small cystic lesions $(<5 \mathrm{~mm}$ ) can be associated with adenomyosis due to minor bleeding into the myometrium, but larger cysts (>1 cm) are rare. In 1908, Cullen [1] first described cystic lesions filled with chocolate-colored fluid in the submucosa of patients with adenomyosis. These cysts were lined with endometrial glands. In 1990, Parulekar [2] first described adenomyotic cysts. These cysts are usually found in younger women with symptoms of chronic pelvic pain and/or dysmenorrhea. Due to the close clinical and radiological similarity of this condition to obstructive Müllerian anomalies, an accurate diagnosis of adenomyotic cysts poses challenges. Evidence from the literature supports surgical excision as the recommended treatment. We report a case of cystic

Received: June 6, 2020 • Revised: October 17, 2020 • Accepted: October 21, 2020 Corresponding author: Sangam Jha

Department of Obstetrics and Gynecology, AllMS Patna, Phulwarisharif, Patna,

801105 Bihar, India

Tel: +91-9827388001 E-mail: sangam.jha78@gmail.com

This is an Open Access article distributed under the terms of the Creative Commons Attribution Non-Commercial License (http://creativecommons.org/licenses/by-nc/4.0/) which permits unrestricted non-commercial use, distribution, and reproduction in any medium, provided the original work is properly cited. adenomyosis that was diagnosed as bicornuate uterus with hematometra in the left horn on ultrasonography. The diagnosis was confirmed via hysteroscopy based on the visualization of two ostia; the presence of a normal uterine cavity ruled out a Müllerian anomaly, and laparoscopy showed a myometrial cyst located on the left cornu of the uterus. The cyst was laparoscopically excised with repair of the defect. The patient recovered well and has been symptom-free since surgery. This case report was written to explore the clinical features and treatment of an adenomyotic cyst in order to help facilitate the early diagnosis and appropriate treatment of this rare entity.

\section{Case report}

This study was performed in accordance with the principles of the Declaration of Helsinki. Ethical approval for this case report was waived by the Institutional Ethical Committee of AlIMS Patna. Informed consent was obtained from the patient for the publication of this report.

A 28-year-old woman (G4P2A2L2) presented with a 1-year history of severe progressive dysmenorrhea. She had experienced menarche at 14 years of age. The patient had no previous history of dysmenorrhea. Pain, localized at the left iliac region, typically started 2-3 days before the onset of menses and persisted for a week afterward. This 
pain was refractory to analgesic drugs. The patient's menstrual cycle was regular (28 days/4-5 days). She had experienced two normal vaginal deliveries and one spontaneous abortion. The patient had a history of suction and evacuation (induced abortion) along with tubal ligation 1 year prior. Her symptoms appeared following that surgical intervention. Otherwise, the patient had no noteworthy past medical history.

Local examination revealed a mildly enlarged uterus with a small, slightly tender mass palpable on the left side of the uterus. Transabdominal ultrasound showed a bicornuate uterus with an anechoic collection measuring $3.3 \times 1.2 \mathrm{~cm}$ in the left horn. The bilateral adnexa were normal (Figure 1). The patient refused magnetic resonance imaging (MRI) due to financial constraints. Thus, under a diagnosis of bicornuate uterus with left-horn hematometra, we proceeded with hysteroscopy and laparoscopic excision of the rudimentary horn. On hysteroscopy, the uterine cavity and the bilateral ostia appeared normal (Figure 2). On laparoscopy, a bulge of $3 \times 3 \mathrm{~cm}$ was seen on the left cornu of the uterus (Figure 3A). The attachments of the bilateral Fallopian tubes and round ligament to the uterus were normal. To make a more precise diagnosis of the cystic collection, needle aspiration was performed, and approximately $7 \mathrm{~mL}$ of chocolate-colored fluid was aspirated from the mass (Figure 3B). The surgical procedure consisted of wide excision of the lesion followed by reconstruction of the defect (Figure 3C and D).

Intrauterine injection of methylene blue during the procedure revealed no communication between the lesion and the endometrial cavity. The patient was discharged on the day after surgery and was prescribed dienogest $(2 \mathrm{mg}$ ) for 3 months. The patient did not experience dysmenorrhea upon menstruation. Biopsy of the lesion revealed a cyst lined by endometrial glands along with stroma and surrounding myometrial hyperplasia. Hemosiderin-laden macrophages were observed in the endometrial tissue. The findings were consistent with an adenomyotic cyst.

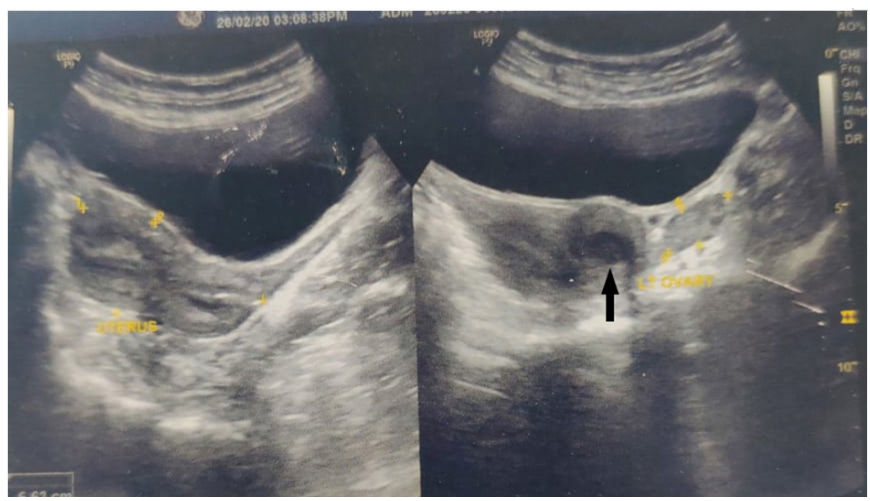

Figure 1. Sonographic finding of a bicornuate uterus with an anechoic collection measuring $3.3 \times 1.2 \mathrm{~cm}$ in the left horn (black arrow).

\section{Discussion}

Adenomyosis is histologically defined as the invasion of the myo-

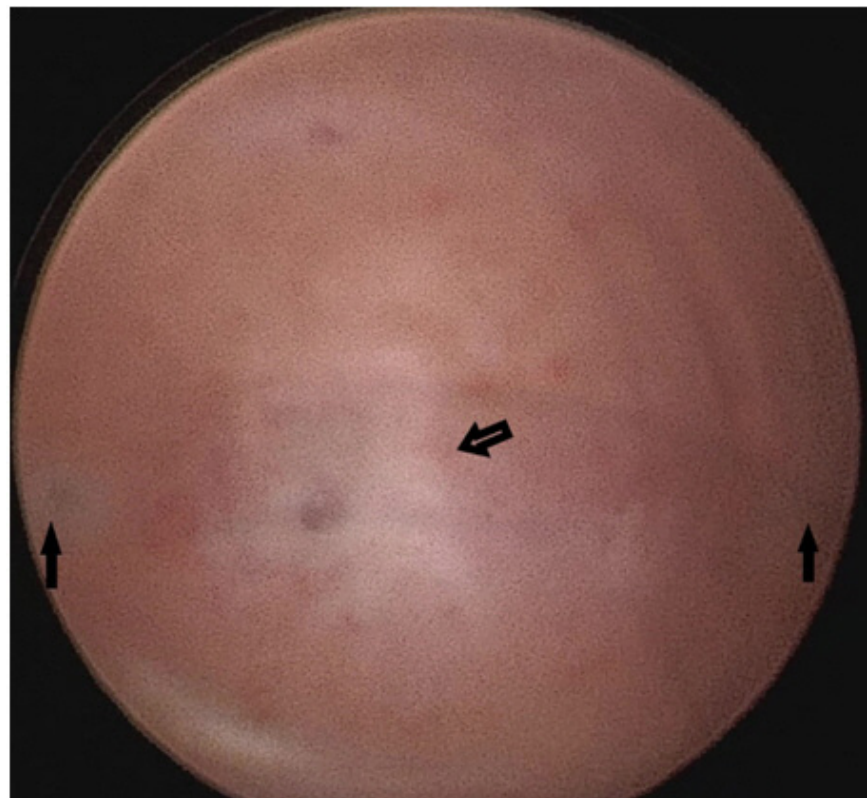

Figure 2. Hysteroscopic view showing bilateral ostia (solid arrows) with mild adhesion at the fundus (hollow arrow).
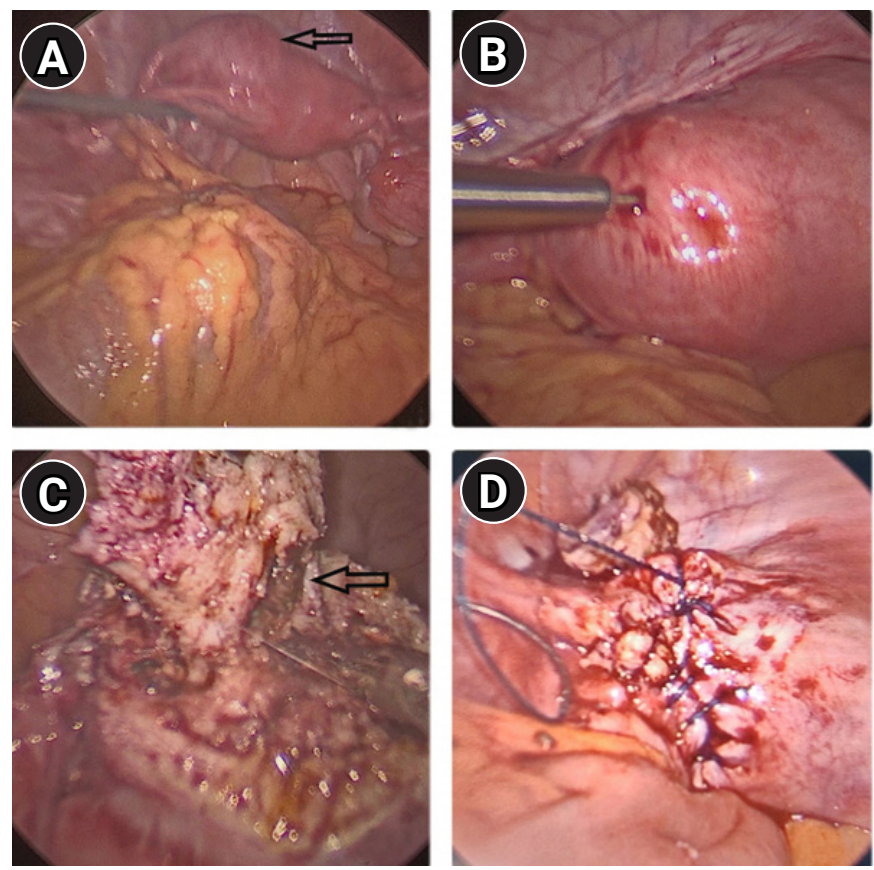

Figure 3. Laparoscopic view of the cystic lesion. (A) Uterus with an anterolateral adenomyotic cyst on the left side of the uterine fundus (arrow). (B) Aspiration of chocolate-colored fluid from the cyst. (C) At the time of cyst excision, a cystic cavity with chocolate-colored fluid was clearly seen (arrow). (D) The uterine myometrium was closed laparoscopically in two layers with continuous sutures. 
metrium by endometrial glands and/or stroma deeper than $2.5 \mathrm{~mm}$ from the endometrial-myometrial junction with accompanying surrounding myometrial hyperplasia. Adenomyosis is clinicohistologically classified into three categories: diffuse, with thickening of the junctional zone and diffuse myometrial involvement; focal, including well-circumscribed adenomyotic masses and cystic adenomyomas; and polypoid adenomyomas, both typical and atypical [3]. In 2015, Brosen et al. [4] classified adenomyotic cysts into five subtypes based on location. Subtype A1 includes submucous or intramural cystic adenomyosis, subtype $A 2$ includes cystic polypoid lesions, subtype B1 includes subserous cystic adenomyosis, subtype B2 includes cases involving exophytic growth, and subtype $C$ comprises uterine-like masses within the uterus also known as juvenile cystic adenomyomas (JCAs) or accessory and cavitated uterine masses. These cysts are lined by ectopic endometrium with thin stroma throughout the cyst, and the surrounding myometrium may exhibit hyperplasia [5]. The cysts are clinically characterized by intractable progressive dysmenorrhea owing to an increase in intracystic pressure as a result of estrogen-dependent cyclical bleeding and shedding of ectopic endometrium within the myometrium. The pathogenesis of these cystic myometrial lesions is uncertain. The juvenile form is considered a congenital anomaly that develops from the proliferation and persistence of Müllerian tissue at or near the insertion of the round ligament on the uterus and likely results from gubernaculum dysfunction [6].

In 2011, Chun et al. [7] proposed these diagnostic criteria for JCA: (1) an age of onset of $<18$ years or severe dysmenorrhea developing within 5 years after the onset of menarche; (2) no history of uterine surgery; and (3) a diameter of the cystic cavity of $>5 \mathrm{~mm}$. The adult form is hypothesized to result from injury to the endometrial-myometrial junction and invagination of the endometrial glands into the myometrium following uterine surgery [8]. However, a few investigators have described JCA as a cystic variant of adenomyosis rather than a congenital anomaly $[9,10]$. Based on the relevant literature, we consider the present case to be an acquired adenomyotic cyst, even though the location of the cyst was typical of JCA.

Diagnosis of this lesion may pose difficulties due to its clinical and radiological similarity to congenital Müllerian anomalies, especially the presence of a non-communicating rudimentary horn with unicornuate uterus or bicornuate uterus with segmental atresia. While adenomyosis is usually asymptomatic, adenomyotic cysts generally present with chronic pelvic pain or progressive dysmenorrhea. Transvaginal sonography is the first-line modality for the evaluation of intractable dysmenorrhea. However, the specific sonographic features of an adenomyotic cyst and its relationship with the endometrial cavity can be challenging to recognize. MRI, as a noninvasive modality with excellent tissue characterization capacity, is very useful for di- agnosing such a lesion as well as differentiating it from complex uterine anomalies [11]. However, cases have been reported in which MRI failed to yield a correct diagnosis, so imaging also has its pitfalls [12]. Unfortunately, in the current case, we could not perform MRI due to financial constraints. Sonography indicated an obstructive Müllerian anomaly, but hysteroscopy was also performed to ascertain the accuracy of this diagnosis. Visualization of two ostia on hysteroscopy ruled out an obstructive Müllerian anomaly.

Zhou et al. [13] reported a case of a giant adenomyotic cyst with a levonorgestrel-containing intrauterine device present in the cyst cavity in a 46-year-old woman. The cyst had developed following myomectomy. The case was managed with laparoscopic excision of the mass along with removal of the intrauterine device. Similar cases have been reported in which patients developed cysts after some form of uterine surgery $[14,15]$, hence supporting the hypothesis of endometrial-myometrial junctional injury as an adenomyotic cyst precursor. In the present case, too, the patient experienced progressive dysmenorrhea following suction and curettage.

The treatment of an adenomyotic cyst involves wide surgical excision, though the symptoms can be temporarily relieved by the use of gonadotropin-releasing hormone, cyclical oral contraceptives, or analgesics. As minimally invasive methods have many advantages, laparoscopy is the route of choice. Hysteroscopic resection of the lesion is the preferred mode of treatment for the submucosal subtype [14]. In the present case, we performed laparoscopic radical excision of the cyst.

To conclude, cystic adenomyosis of the uterus is rare. Diagnosis poses a challenge, as this condition is usually misdiagnosed as an obstructive Müllerian anomaly, a degenerated myoma, or (rarely) as an adnexal cyst. Previous uterine surgery and injury to the endometrial-myometrial junction are precursors of the disease pathology. Radical excision of the lesion is the definitive and preferred mode of treatment. No clinical data are available on whether postoperative medication can effectively prevent recurrence of this condition. Thus, research is urgently required regarding how to effectively detect and treat intrauterine cystic adenomyosis and to develop methods to prevent its recurrence.

\section{Conflict of interest}

No potential conflict of interest relevant to this article was reported.

\section{ORCID}

Sangam Jha

https://orcid.org/0000-0002-4349-1589 


\section{References}

\section{Cullen TS. Adenomyoma of the uterus. Philadelphia: Saunders;} 1908.

2. Parulekar SV. Cystic degeneration in an adenomyoma (a case report). J Postgrad Med 1990;36:46-7.

3. Grimbizis GF, Mikos T, Tarlatzis B. Uterus-sparing operative treatment for adenomyosis. Fertil Steril 2014;101:472-87.

4. Brosens I, Gordts S, Habiba M, Benagiano G. Uterine cystic adenomyosis: a disease of younger women. J Pediatr Adolesc Gynecol 2015;28:420-6.

5. Takeda A, Sakai K, Mitsui T, Nakamura H. Laparoscopic management of juvenile cystic adenomyoma of the uterus: report of two cases and review of the literature. J Minim Invasive Gynecol 2007;14:370-4.

6. Acien P, Bataller A, Fernandez F, Acien Ml, Rodriguez JM, Mayol MJ. New cases of accessory and cavitated uterine masses (ACUM): a significant cause of severe dysmenorrhea and recurrent pelvic pain in young women. Hum Reprod 2012;27:683-94.

7. Chun SS, Hong DG, Seong WJ, Choi MH, Lee TH. Juvenile cystic adenomyoma in a 19-year-old woman: a case report with a proposal for new diagnostic criteria. J Laparoendosc Adv Surg Tech A 2011;21:771-4.

8. Tan J, Yong P, Bedaiwy MA. A critical review of recent advances in the diagnosis, classification, and management of uterine adenomyosis. Curr Opin Obstet Gynecol 2019;31:212-21.

9. Takeuchi H, Kitade M, Kikuchi I, Kumakiri J, Kuroda K, Jinushi M. Diagnosis, laparoscopic management, and histopathologic findings of juvenile cystic adenomyoma: a review of nine cases. Fertil Steril 2010;94:862-8.

10. Dogan E, Gode F, Saatli B, Secil M. Juvenile cystic adenomyosis mimicking uterine malformation: a case report. Arch Gynecol Obstet 2008;278:593-5.

11. Krentel H, Cezar C, Becker S, Di Spiezio Sardo A, Tanos V, Wallwiener $M$, et al. From clinical symptoms to MR imaging: diagnostic steps in adenomyosis. Biomed Res Int 2017;2017:1514029.

12. Dadhwal V, Sharma A, Khoiwal K. Juvenile cystic adenomyoma mimicking a uterine anomaly: a report of two cases. Eurasian $J$ Med 2017;49:59-61.

13. Zhou Y, Chen ZY, Zhang XM. Giant exophytic cystic adenomyosis with a levonorgestrel containing intrauterine device out of the uterine cavity after uterine myomectomy: a case report. World J Clin Cases 2020;8:188-93.

14. Fan YY, Liu YN, Li J, Fu Y. Intrauterine cystic adenomyosis: report of two cases. World J Clin Cases 2019;7:676-83.

15. Kim MJ. A case of cystic adenomyoma of the uterus after complete abortion without transcervical curettage. Obstet Gynecol Sci 2014:57:176-9. 